\title{
Non-histone nuclear factor HMGB1 is phosphorylated and secreted in colon cancers
}

\author{
Hyun Ju Kang ${ }^{1,2, *}$, Hanna Lee ${ }^{1,2, *}$, Hee-Jung Choi ${ }^{1,2}$, Ju Ho Youn ${ }^{2,3}$, Jeon-Soo Shinn ${ }^{2,3}$, Yeong Hee Ahn ${ }^{4}$, \\ Jong Shin $\mathrm{YoO}^{4}$, Young-Ki Paik ${ }^{5}$ and Hoguen Kim ${ }^{1,2}$
}

The high mobility group box 1 (HMGB1) protein, a non-histone nuclear factor, is overexpressed and localizes to the cytoplasm in some cancer cells. However, the mechanism of cytoplasmic HMGB1 transport, extracellular secretion, and its role in cancer progression is not clear. To simulate the activated state of HMGB1, we mutated serine residues of nuclear localization signals (NLSs) to glutamic acid and performed transfection assays. We carried out a kinase inhibitor study and evaluated the cell migration by invasion assay. We showed that phosphorylated HMGB1 localizes in the cytoplasm of colon cancer cells and also showed the interaction of PKC and HMGB1 by immunoprecipitation analysis. Concurrent mutations at six serine residues $(35,39,42,46,53$, and 181) to glutamic acid induced the nuclear to cytoplasmic transport of HMGB1, which was detected in the culture medium. We also observed that the secretion of HMGB1 correlated with increased cancer cell invasiveness. Our results suggest that phosphorylated HMGB1 is transported to the cytoplasm, is subsequently secreted from the cell, and has a role in tumor progression through the activation of genes related to cell migration.

Laboratory Investigation (2009) 89, 948-959; doi:10.1038/labinvest.2009.47; published online 8 June 2009

KEYWORDS: HMGB1 phosphorylation; HMGB1 secretion; colon cancer cells; PKC; invasion

The high mobility group box 1 (HMGB1) protein is one of several non-histone chromosomal proteins found in eukaryotic cells. The protein is typically located in the nucleus where it binds to minor groove DNA, promotes the assembly of site-specific DNA-binding factors, and is involved in gene transcription. ${ }^{1-3}$ In addition to transcriptional regulation, HMGB1 has several well-established extracellular roles. HMGB1 localizes to the cell membrane of neurites for outgrowth. ${ }^{4}$ It is secreted from activated monocytes, macrophages, and NK cells, and acts extracellularly as a proinflammatory cytokine. ${ }^{5}$ It is passively secreted by necrotic cells, although not by apoptotic cells, and triggers inflammation. ${ }^{6,7}$ Recent studies have shown that the posttranslational modification status of HMGB1 is related to translocation within cells and secretion by inflammatory cells, in which it shuttles between the nucleus to the cytoplasm through hyperacetylation and phosphorylation in macrophages, and is monomethylated at Lys42 in neutrophils. ${ }^{1,2,8,9}$ Cytosolic HMGB1 accumulates and is secreted through a vesicle-mediated secretory pathway in monocytic cells. $^{10,11}$

One important extracellular role of HMGB1 is the promotion of cell migration and metastasis. ${ }^{7,12,13}$ We and others have previously described the overexpression and cytoplasmic localization of HMGB1 in some tumor cells. ${ }^{14,15}$ These observations raise several additional questions, such as: (1) How is HMGB1 in tumor cells transported from the nucleus to the cytoplasm? (2) If HMGB1 is transported to the cytoplasm and subsequently secreted from cancer cells, which signal triggers its movement? (3) If HMGB1 is secreted, does it contribute to cancer cell migration and metastasis?

In this report, we address these questions and show that phosphorylated HMGB1 localizes in the cytoplasm of colon cancer cells and is secreted in the extracellular space. We also showed the secreted HMGB1 induces the MMP pathway and increases the invasiveness of these cancer cells. These findings suggest an autocrine and/or paracrine role of HMGB1 in cancer progression.

\footnotetext{
${ }^{1}$ Department of Pathology, Yonsei University College of Medicine, Seoul, Korea; ${ }^{2}$ Brain Korea 21 Project for Medical Sciences, Yonsei University College of Medicine, Seoul, Korea; ${ }^{3}$ Department of Microbiology, Yonsei University College of Medicine, Seoul, Korea; ${ }^{4}$ Division of Instrument Development, Korea Basic Science Institute, Daejeon, Republic of Korea and ${ }^{5}$ Yonsei Proteome Research Center, Department of Biochemistry and Bioproducts Research Center, Yonsei University, Seoul, Korea Correspondence: Professor H Kim, MD, Department of Pathology, Yonsei University College of Medicine, CPO Box 8044, Seoul, Korea 120-1752. E-mail: hkyonsei@yuhs.ac

${ }^{*}$ These two authors contributed equally to this work.

Received 19 September 2008; revised 2 April 2009; accepted 28 April 2009
} 


\section{MATERIALS AND METHODS}

\section{Cell Lines and Tissue Samples}

Cell lines were obtained from the ATCC (American Type Culture Collection) (http://www.atcc.org) or from the KCLB (Korean Cell Line Bank) (http://cellbank.snu.ac.kr). Colo205, DLD-1, HCT116, HCT8, HT29, Ls174T, NCIH508, NCIH747, SNU-C4, SNU-C2A, and SW480 cells were grown in the RPMI or DMEM medium supplemented with $10 \%$ fetal bovine serum (Life Technologies Inc., Grand Island, NY, USA), at $37^{\circ} \mathrm{C}$ in the presence of $5 \% \mathrm{CO}_{2}$. CCD-18Co and RKO cells were cultured in the minimal essential medium supplemented with $10 \%$ fetal bovine serum.

Eight colorectal carcinomas and matched normal mucosa were included in this study. All cases were identified in the Department of Pathology at the Yonsei University Medical Center between September 1995 and November 2000. Some of the fresh specimens were supported by the Liver Cancer Specimen Bank from the National Research Resource Bank Program of the Korea Science and Engineering Foundation of the Ministry of Science and Technology. Authorization for the use of these tissues for research purposes was obtained from the Institutional Review Board of the Yonsei University College of Medicine. To enrich the tumor cell population, areas with more than $90 \%$ tumor cells were selected from hematoxylin-eosin-stained slides using the cryostat microdissection technique.

\section{Immunohistochemical Analysis}

Formalin-fixed and paraffin-embedded tissues were used for the immunostaining of HMGB1. Deparaffinization and rehydration were performed using xylene and alcohol. The sections were treated with $0.3 \%$ hydrogen peroxidase for $3 \mathrm{~min}$ and blocking antibody for $30 \mathrm{~min}$. The antibody against HMGB1 was at 1:100 (v/v). The avidin-biotin complex methodology was used. The chromogen was diaminobenzidine and counterstaining was performed with methyl green.

\section{Protein Extraction}

Tumor tissues and matched non-tumorous tissues were suspended in an ice-cold lysis buffer (50 mM Tris ( $\mathrm{pH} 7.4$ ), $1 \%$ Triton X-100, $5 \mathrm{mM}$ EDTA, $1 \mathrm{mM} \mathrm{KCl}, 140 \mathrm{mM} \mathrm{NaCl}$, $2 \mathrm{mM} \mathrm{MgCl}_{2}, 1 \mathrm{mM}$ phenylmethylsulfonyl fluoride, $1 \mathrm{mM}$ sodium fluoride, $1 \%$ aprotinin, $1 \mu \mathrm{M}$ leupeptin, and $1 \mathrm{mM}$ sodium orthovanadate) for $15 \mathrm{~min}$. Suspensions were sonicated for $\sim 30 \mathrm{~s}$ and centrifuged at $20000 \times g$ for $15 \mathrm{~min}$.

Four samples with sufficient material were selected for nuclear and cytoplasmic protein fractionation. Briefly, $\sim 0.3 \mathrm{mg}$ of frozen tissue was added to $1 \mathrm{ml}$ of buffer A (10 mM HEPES ( $N$-2-hydroxyethylpiperrazine- $N$-2-ethanesulfonic acid) (pH 7.9), $10 \mathrm{mM} \mathrm{KCl,} 0.1 \mathrm{mM}$ EDTA, $0.1 \mathrm{mM}$ EGTA, $1 \mathrm{mM}$ dithiothreitol, and $0.5 \mathrm{mM}$ phenylmethylsulfonyl fluoride) and homogenized. The cells were allowed to swell on ice for $15 \mathrm{~min}$, after which $50 \mu \mathrm{l}$ of $10 \% \mathrm{NP}-40$ was added. After vigorous vortex for $10 \mathrm{~s}$, the homogenate was centrifuged for $30 \mathrm{~s}$. The supernatant was collected and used for cytoplasmic protein assays. The nuclear pellet was suspended in $100 \mu \mathrm{l}$ of ice-cold buffer C (20 mM HEPES (pH 7.9), $0.4 \mathrm{mM} \mathrm{NaCl}, 1 \mathrm{~mm}$ EDTA, $1 \mathrm{mM}$ EGTA, $1 \mathrm{mM}$ dithiothreitol, and $1 \mathrm{mM}$ phenylmethylsulfonyl fluoride) and shaken at $4{ }^{\circ} \mathrm{C}$ for $15 \mathrm{~min}$. Samples were then centrifuged for $5 \mathrm{~min}$, and the supernatant was collected and used for nuclear protein assays.

\section{Immunoblot and Immunoprecipitation Analysis}

Total protein lysate of $20 \mu \mathrm{g}$ of and $40 \mu \mathrm{g}$ of cytoplasmic extracts, and $5 \mu \mathrm{g}$ of nuclear protein extracts were fractionated by SDS-PAGE. Proteins in the gel were transferred and blocked with TBS-T containing 5\% skim milk. Anti-HMGB1 (BD Biosciences, Franklin Lakes, NJ, USA), anti-hnRNPC (Santa Cruz Biotechnology, Delaware Avenue, CA, USA), anti-pan acetylation (Santa Cruz Biotechnology), anti-phosphoserine (BD Biosciences), anti-GAPDH (glyceraldehyde-3phosphate dehydrogenase) (Trevigen, Gaithersburg, MD, USA), anti-PKC (Santa Cruz Biotechnology), and anti- $\alpha$ Tubulin (Oncogene, Cambridge, MA, USA) were diluted 1:5000 in the blocking buffer and incubated for $1 \mathrm{~h}$ at room temperature. Membranes were washed and then incubated for $1 \mathrm{~h}$ with HRP-conjugated secondary antibody (Santa Cruz Biotechnology), washed, and developed with ECL-Plus (Amersham Pharmacia Biotech, Uppsala, Sweden). For immunoprecipitation experiments, $500 \mu \mathrm{g}$ of tissue lysates were pre-cleared with PBS and gently rocked on an orbital shaker with anti-HMGB1 (BD Biosciences) at $4{ }^{\circ} \mathrm{C}$. The immune complexes were collected by centrifugation and boiled to dissociate the immunocomplexes from the beads. The beads were collected by centrifugation and protein separation was performed by SDS-PAGE with the supernatant fraction.

\section{2-Dimensional Electrophoresis (2DE) Immunoblot Analysis}

Colorectal carcinoma and matched normal tissues were suspended in the sample buffer $(40 \mathrm{mM}$ Tris, $7 \mathrm{M}$ urea, $2 \mathrm{M}$ thiourea, 4\% CHAPS, and $100 \mathrm{mM} \mathrm{1,4-dithioerythritol)}$ and in a protease inhibitor cocktail (Roche, Mannheim, Germany). Suspensions were sonicated for $\sim 30 \mathrm{~s}$ and centrifuged at $100000 \times g$ for $45 \mathrm{~min}$. One milligram of total protein was used for each $2 \mathrm{DE}$ analysis. Aliquots of proteins in the sample buffer were applied onto immobilized $\mathrm{pH} 3-10$ non-linear gradient strips (Amersham Pharmacia Biotech), and isoelectric focusing was conducted at $100000 \mathrm{Vh}$. 2DE was performed in $9-16 \%$ linear gradient polyacrylamide gels $(18 \mathrm{~cm} \times 20 \mathrm{~cm} \times 1.5 \mathrm{~mm})$ as previously described, ${ }^{16}$ transferred into PVDF membranes, and subsequently blocked in TBS-T containing 5\% skim milk. Anti-HMGB1 (BD Biosciences) was diluted 1:5000 in the blocking buffer and incubated with the membranes for $1 \mathrm{~h}$ at room temperature. After washing, the membranes were incubated for $1 \mathrm{~h}$ 
with HRP-conjugated secondary antibody (Santa Cruz Biotechnology), washed, and developed with ECL-Plus (Amersham Pharmacia Biotech).

\section{Vector Construction and Transfection}

We performed transfection assays with the following four vectors (kindly provided by Dr J-S Shin, Department of

a Vector structure

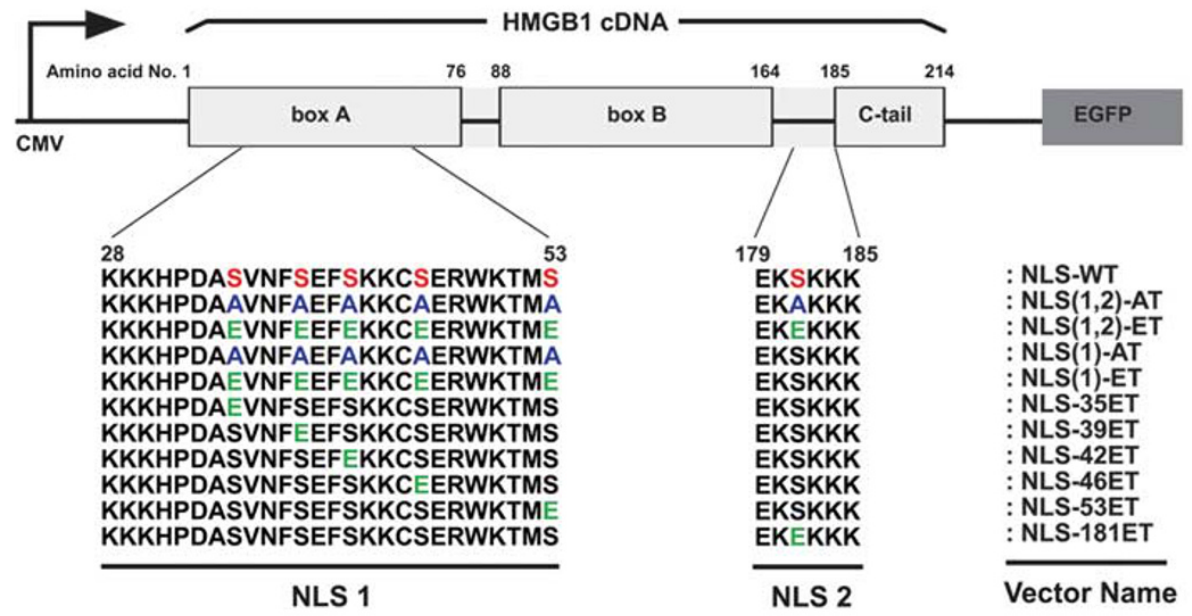

b NLS-WT
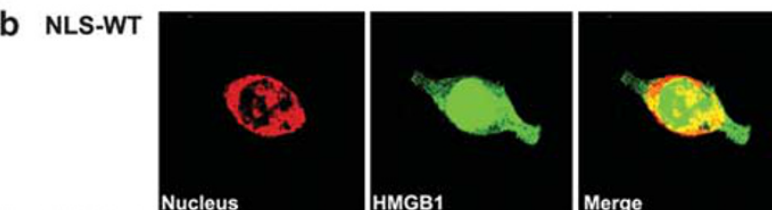

NLS(1,2)-AT
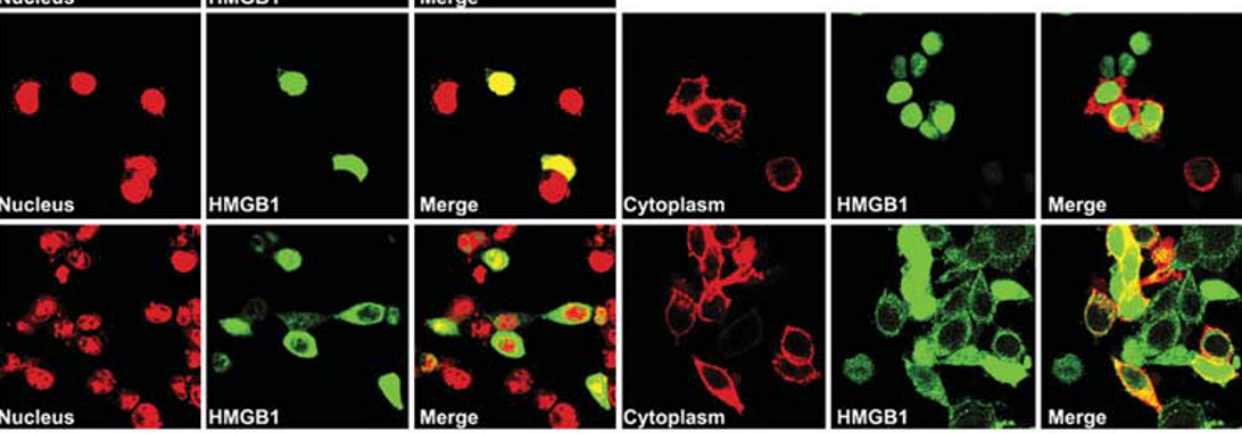

C NLS(1)-AT
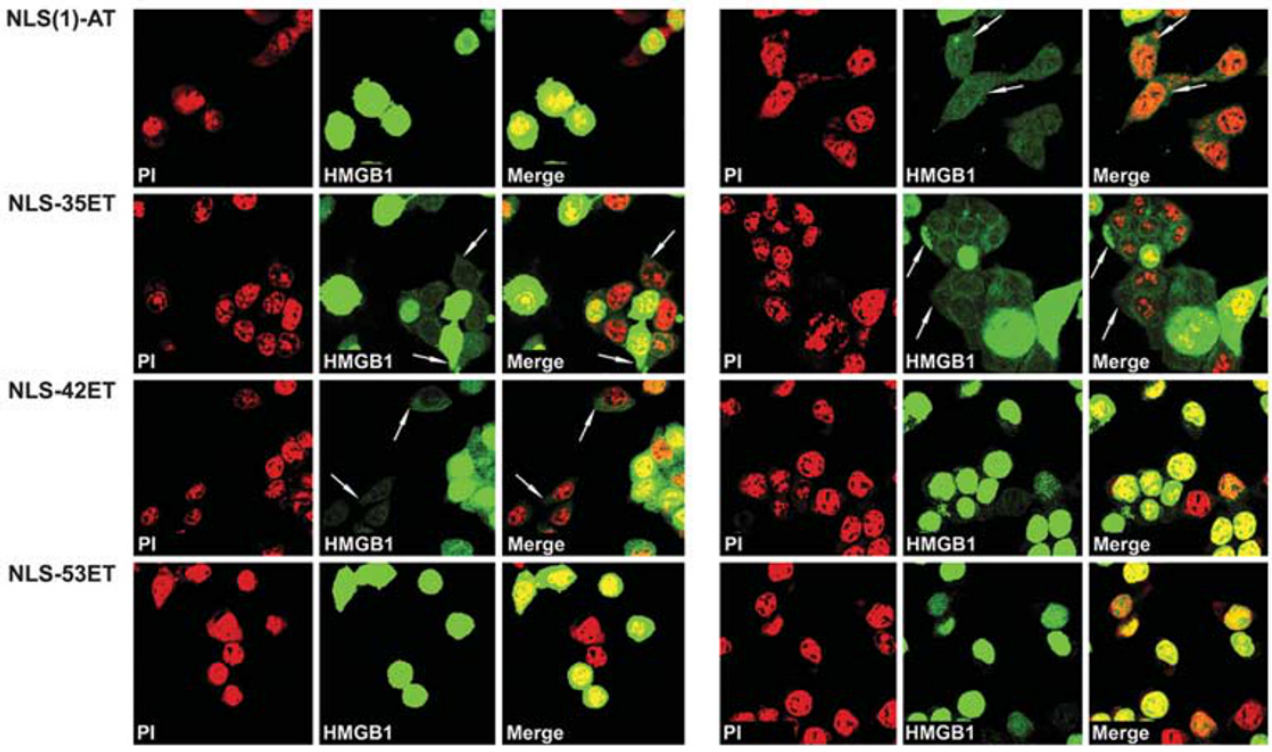

NLS-39ET 
Microbiology, Yonsei University College of Medicine, Korea): (1) EGFPN1, used as a control; (2) nuclear localization signal (NLS)-WT, containing wild-type HMGB1; (3) NLS(1,2)-AT, containing HMGB1 sequence in which the six serine residues in the NLS 1 and 2 regions were mutated to alanine for the inhibition of HMGB1 phosphorylation; and (4) NLS(1,2)ET, containing HMGB1 sequence in which the six serine residues in the NLS 1 and 2 regions were mutated to glutamic acid for simulation of phosphorylated HMGB1. ${ }^{17}$

Six vectors, namely NLS-35ET, NLS-39ET, NLS-42ET, NLS-46ET, NLS-53ET, and NLS-181ET that contained serine to glutamic acid mutations in the NLS1 and NLS2 regions were constructed. One vector (NLS(1)-ET) contained serine to glutamic acid mutations of all five serine residues in the NLS1 region. An additional vector (NLS(1)-AT) contained serine to alanine mutations in all five serine residues. All the vectors were constructed using the QuikChange SiteDirected Mutagenesis Kit (Stratagene, La Jolla, CA, USA), and sequences were confirmed by direct sequencing analysis (Figure 1a).

HCT116 cell and CCD-18Co were transiently transfected using Lipofectamine 2000 (Invitrogen, Carlsbad, CA, USA) with the constructed plasmid in LabTek II chambers (Nalgene, Waltham, MA, USA) or in a $60-\mathrm{mm}$ tissue culture plate. Cells were observed or harvested 2 days later.

\section{Immunofluorescence Imaging Analysis}

Cells were cultured in LabTek II chambers (Nalgene) and fixed in 10\% paraformaldehyde in PBS for $10 \mathrm{~min}$ at room temperature. The cells were then washed with PBS and incubated for $5 \mathrm{~min}$ at $4{ }^{\circ} \mathrm{C}$ with the permeabilization buffer containing $0.1 \%$ Triton X-100 in PBS. Samples were blocked with $2 \%$ BSA in PBS for 30 min and incubated with rabbit anti-HMGB1 for $1 \mathrm{~h}$ at room temperature. After three washes with $0.2 \%$ BSA in PBS, Alexa Fluor 488 secondary antibody (Invitrogen) was added. The mounting medium containing propidium iodide (Vector Laboratories, Burlingame, CA, USA) was used. Fluorescently labeled cells were observed using the Zeiss LSM 510 confocal microscope (Carl Zeiss, Germany), and images were processed using the LSM 5 Image Examiner software.

\section{Drug Treatment}

HCT116 cell lines were treated with kinase inhibitors Quercetin, Tamoxifen, Myricetin (Calbiochem, Dermstadt, Germany), Rottlerin (Sigma-Aldrich, St Louis, MO, USA), and chromosome region maintenance 1 (CRM1)-mediated active export inhibitor Leptomycine B (Sigma-Aldrich). We treated with Myricetin at $3 \mu \mathrm{M}$ or $10 \mu \mathrm{M}$, Quercetin at $20 \mu \mathrm{M}$, Tamoxifen at $6 \mu \mathrm{M}$, and Rottlerin at $10 \mu \mathrm{M}$. All drugs were dissolved in either distilled water or DMSO (dimethyl sulfoxide) and were added to the culture medium in volumes not exceeding $0.5 \%$ of the total culture medium volume.

\section{Invasion Assay}

The Cell Invasion Assay Kit (Chemicon International, Temecula, CA, USA) was used according to the manufacturer's instructions. Briefly, the ECM layer was rehydrated for $1-2 \mathrm{~h}$ at room temperature using the serum-free medium. A volume of $500 \mu \mathrm{l}$ of the medium containing $10 \%$ fetal bovine serum was added to the lower chamber, and prepared cell suspension in the serum-free medium was added to the upper chamber. Cells were incubated for $40 \mathrm{~h}$ at $37^{\circ} \mathrm{C}$ in the presence of $5 \% \mathrm{CO}_{2}$. Non-invading cells were removed from the upper chamber and invasive cells on the lower surface of the membrane were stained for $20 \mathrm{~min}$. Invasiveness quantities were measured by dissolving stained cells in 10\% acetic acid; a consistent amount of the dye-solute mixture was transferred into a 96-well plate for colorimetric reading of $\mathrm{OD}$ at $560 \mathrm{~nm}$. Each experiment was performed in triplicate.

\section{RESULTS \\ Overexpression and Cytoplasmic Localization of HMGB1 in Colon Cancer Cells}

We first examined the amount of HMGB1 in eight colon cancer tissue samples and in normal mucosal tissues by immunoblot analysis. We found that HMGB1 was overexpressed in all colon cancer tissues that were tested. We also observed strong HMGB1 expression in 12 colon cancer cell lines, despite the variability between samples (Figure 2a). We then examined the level of nuclear and cytoplasmic HMGB1 by the fractionation of nuclear and cytoplasmic proteins in four colon cancer tissues and normal mucosal tissues. The normal colonic fibroblast cell line, CCD-18Co, was used as a control. Strong HMGB1 expression was observed in both the nucleus and the cytoplasm in all four colorectal carcinomas and in four colon cancer cell lines. The amount of nuclear HMGB1 in cancer cells, normal tissues, and normal fibroblast cell lines was not significantly different. However, cytoplasmic HMGB1 was absent or was present at low levels in normal tissues and normal fibroblast cell lines (Figure $2 \mathrm{~b}$ and Figure 3), whereas cytoplasmic HMGB1 was found at high levels in tumor cells.

Figure 1 Phosphorylation of serines 35, 39, and 42 is critical for cytoplasmic localization of HMGB1. (a) Schematic diagram of the HMGB1 gene structure and constructed vectors. HMGB1 consists of two DNA-binding domains, the HMG boxes A and B, and an acidic C-terminal domain. The A box and B box both contain nuclear localization signals (NLSs) NLS1, which includes amino acids 28-53, and NLS2, which includes amino acids 179-185. A description of each vector can be found in the text. (b) Localization of HMGB1 in HCT116 cells transfected with NLS-WT, NLS(1,2)-AT, or NLS(1,2)-ET. Cytoplasmic HMGB1 was evident in the cell lines transfected with NLS-WT and NLS(1,2)-ET. (c) Cytoplamic HMGB1 (arrows) is found in the HCT116 cells transfected with NLS(1)-ET (vector for which all five serine residues in the NLS1 region were mutated to glutamic acid), NLS-35ET, NLS-39ET, and NLS-42ET (containing a serine to glutamic acid mutation at the indicated residue; Figure a). 
a
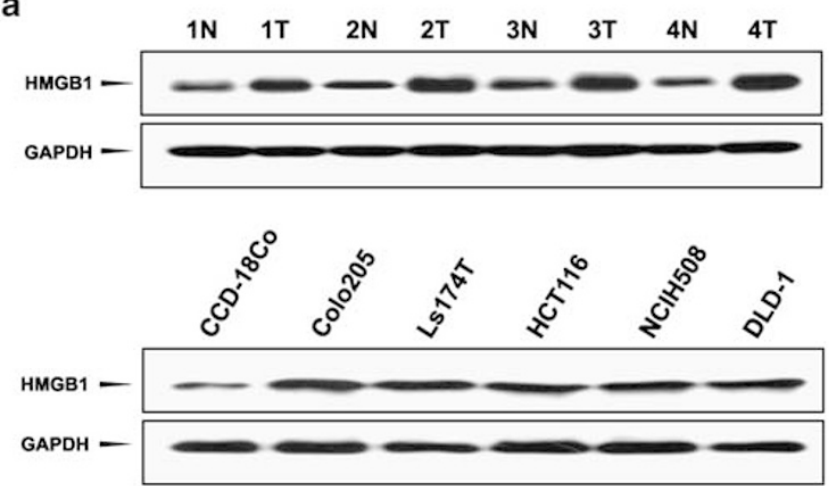

b
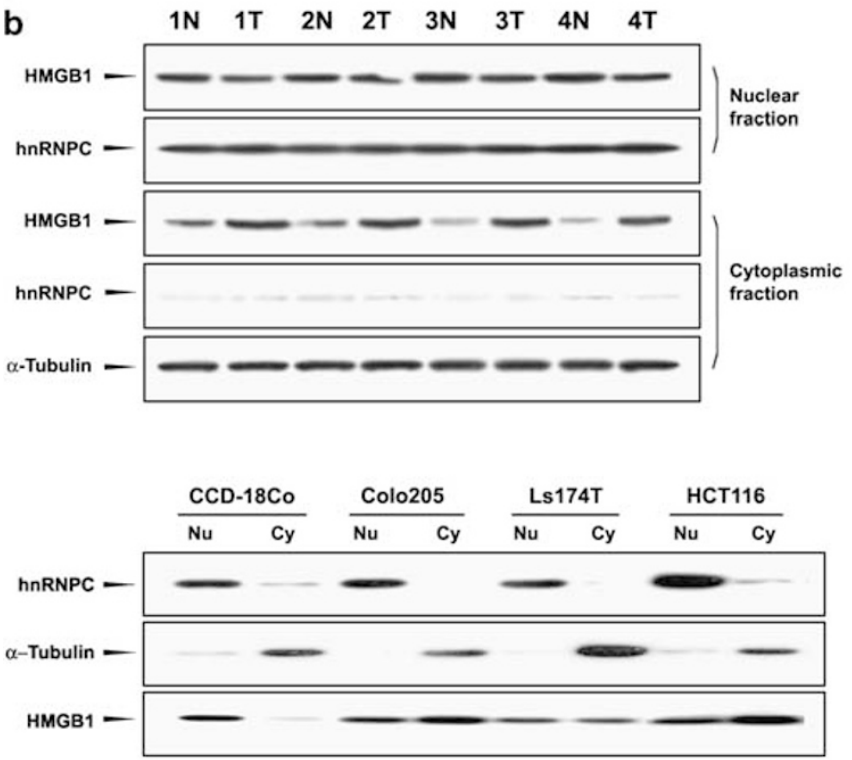
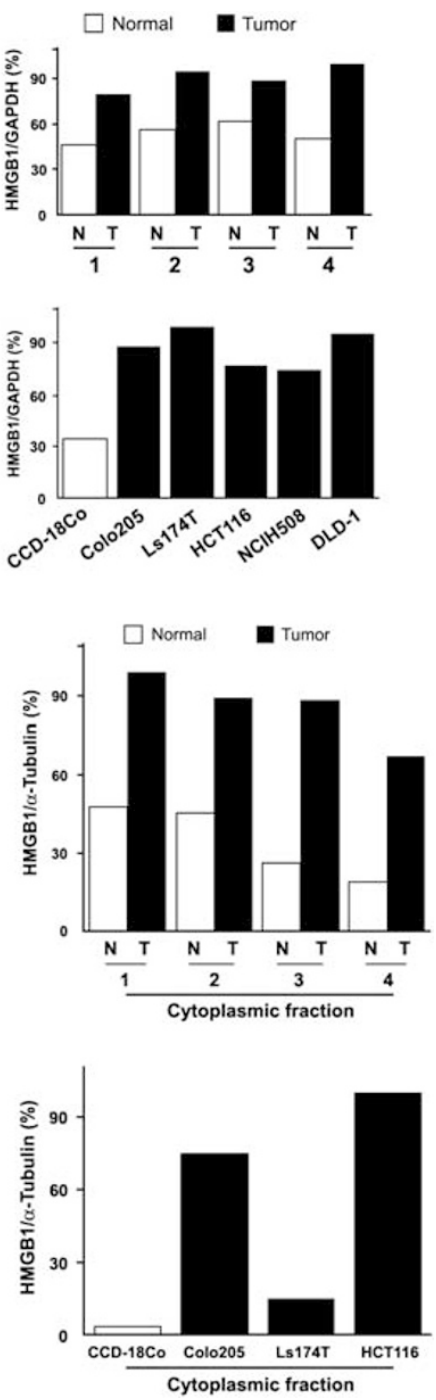

Figure 2 Overexpression and nuclear and cytoplasmic localization of HMGB1 in colon cancer cells. (a) HMGB1 expression in total protein lysates from four colon cancer samples, matched normal mucosal tissue samples, and 12 colon cancer cell lines. $\mathrm{N}$, total protein lysate from the normal colon tissue; $\mathrm{T}$, total protein lysate from the cancer tissue. (b) Analysis of HMGB1 expression in the nuclear and cytoplasmic protein fractions from four colon cancer tissues and five cell lines. Anti-hnRNPC was used for a nuclear protein control. Anti-tubulin was used for a cytoplasmic protein control. Nu, nuclear protein lysates; Cy, cytoplasmic protein lysates.

\section{Identification of Cytoplasmic Phosphorylated HMGB1 in Cancer Cells}

As we observed that the intracellular distribution of HMGB1 differs between tumor and normal mucosa, we next compared the modification status of HMGB1 in these samples by 2DE and an immunoblot analysis using an HMGB1 antibody. Some spots with strong intensities in colon cancer samples had more acidic isoeletric points compared with those from the normal mucosa (Figure 4a). We next used prediction servers (http://www.cbs.dtu.dk/services/NetPhos/ and http:// www.cbs.dtu.dk/services/NetAcet/) to predict the modification status and found that phosphorylation and acetylation may occur in HMGB1. To validate any potential phosphorylation and acetylation modifications in HMGB1, we performed immunoprecipitation assays using protein lysates and an HMGB1 antibody followed by immunoblot analysis using antibodies against phosphorylated and acetylated proteins. We found that cells from both normal mucosa and cancer tissues contained acetylated HMGB1, but phosphorylated HMGB1 was only present in cancer cells (Figure $4 \mathrm{~b}$ ).

\section{Cytoplasmic Transport of HMGB1 is Related to the Modification of Serine Residues in the NLS Sequence}

Some reports have previously suggested that modified HMGB1 is related to its cytoplasmic transport and secretion in inflammatory cells. In this study, we hypothesized that phosphorylation of HMGB1 is required for cytoplasmic transport in cancer cells. To test this hypothesis, we performed transfection assays with control and modified HMGB1 vectors to simulate the phosphorylation state of 
CCD-18Co

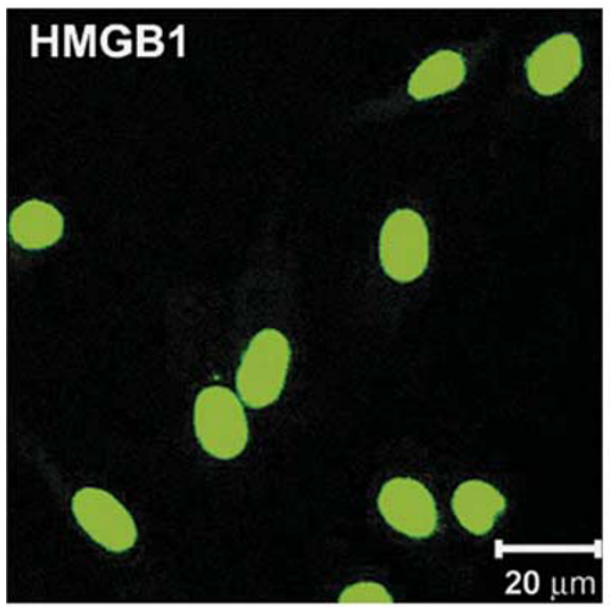

HCT116

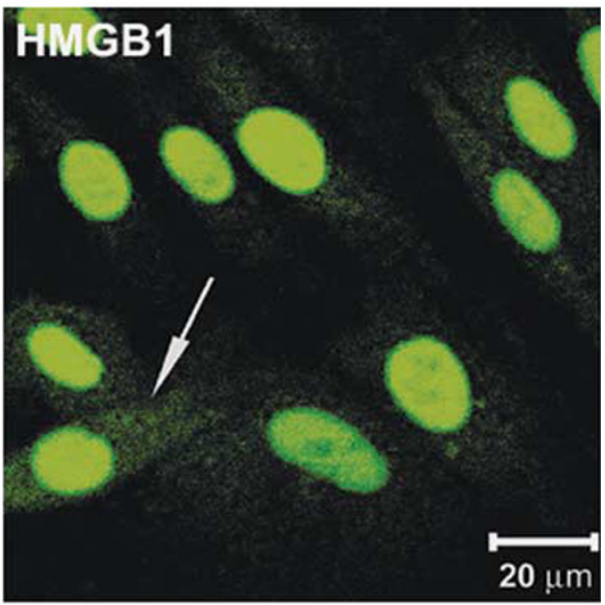

\section{Normal colonic mucosa}
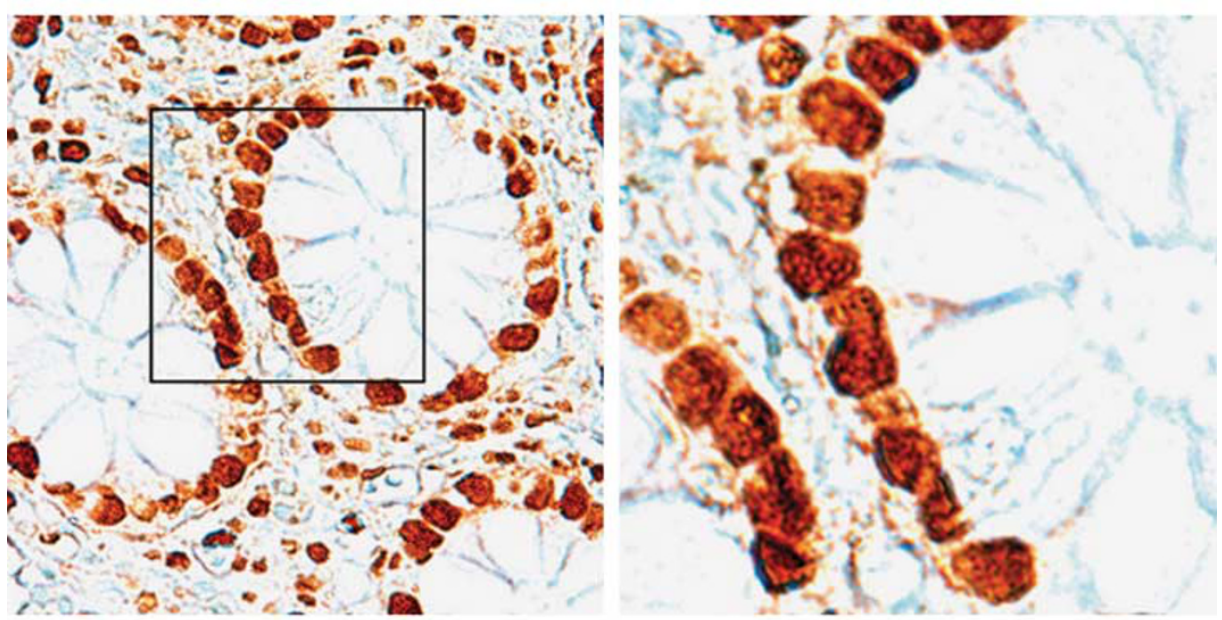

\section{Colon cancer tissue}
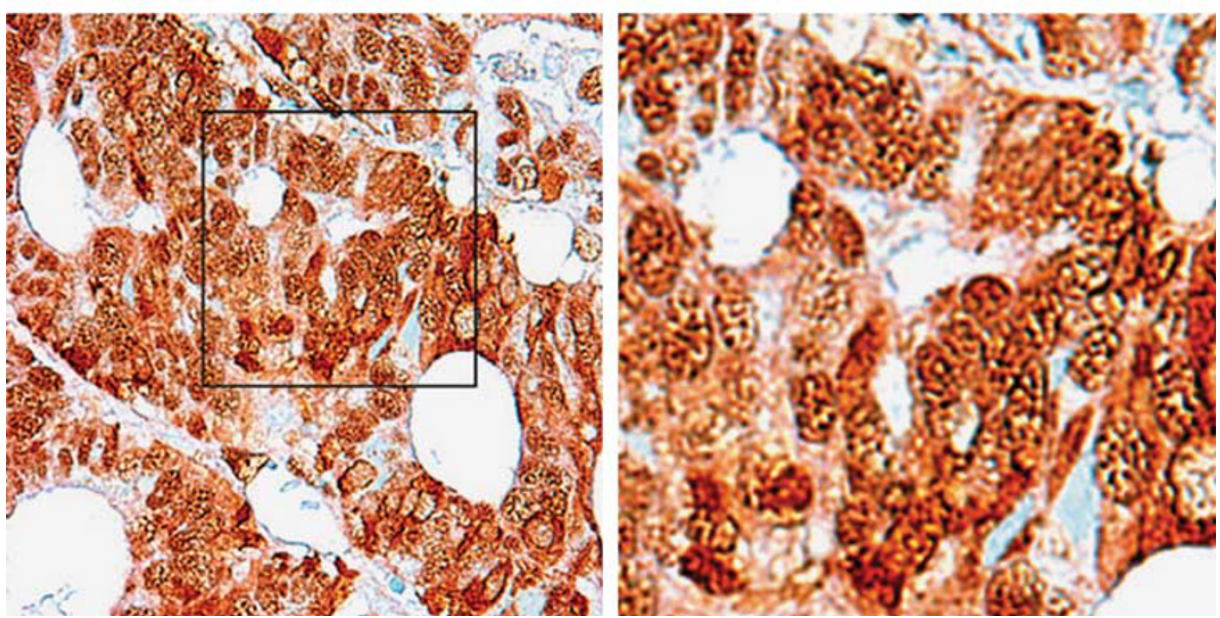

Figure 3 Localization of HMGB1 in CCD-18Co (normal), HCT116 (cancerous) cell lines, normal colonic mucosa, and colon cancer tissues. Both nuclear and cytoplasmic localizations (arrow) were evident in the HCT116 cell line. In normal mucosa, only mucosa localization was evident in colonic epithelial cells. In cancer cells, both nuclear and cytoplasmic localization were evident. 

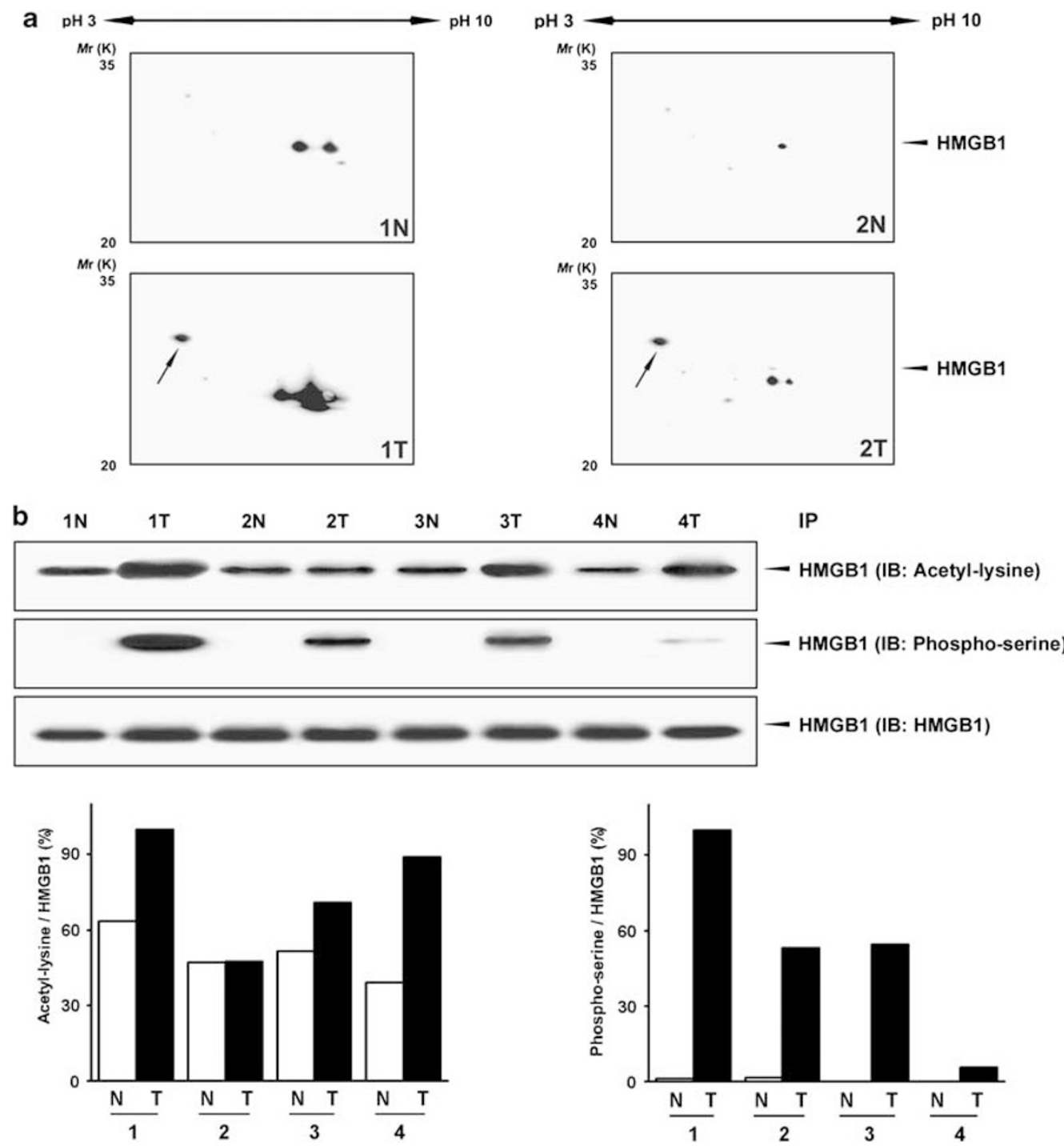

Figure 4 Identification of cytoplasmic phosphorylated HMGB1 in cancer cells. (a) 2DE immunoblot analysis with anti-HMGB1. Some HMGB1 spots with strong intensity in colon cancers show more acidic isoeletric points compared with normal mucosa (arrows). (b) Immunoprecipitation and immunoblot analysis with anti-acetylation and anti-phosphoserine. Both normal mucosa and cancer tissues contained acetylated HMGB1, but phosphorylated HMGB1 was only present in cancerous cells.

translocated HMGB1. We mutated serine residues within both of the nuclear localization signals (NLSs) in HMGB1 to glutamic acid to simulate the phosphorylation status of the protein. We used the following four vectors in this experiment: (1) EGFPN1, used as a negative control; (2) NLS-WT, contains wild-type HMGB1 for overexpression of HMGB1; (3) NLS(1,2)-AT, contains HMGB1 sequence in which six serine residues in NLS 1 and 2 were mutated to alanine for the inhibition of HMGB1 phosphorylation; and (4) NLS(1,2)-ET, contains HMGB1 sequence in which the six serine residues in NLS 1 and 2 were mutated to glutamic acid for the simulation of phosphorylated HMGB1 (Figure 1a). When CCD-18Co cells were transfected with NLS(1,2)-AT or NLS-WT, HMGB1 was observed only in the nucleus. In contrast, high levels of HMGB1 were observed in the cyto- plasm of CCD-18Co cells transfected with the NLS(1,2)-ET vector (Supplementary Figure 1). In similar experiments using the colon cancer cell line HCT116, both nuclear and cytoplasmic HMGB1 was identified after transfection with NLS-WT, and HMGB1 was exclusively located in the nucleus after transfection with the NLS(1,2)-AT vector. In contrast, the majority of HMGB1 was located in the cytoplasm of HCT116 cells transfected with the NLS(1,2)-ET vector (Figure $1 \mathrm{~b}$ ). These results were confirmed by the immunoblot of nuclear and cytoplasmic lysates (data not shown).

\section{Phosphorylation of Serines 35, 39, and 42 are Critical for Nucleus To Cytoplasmic HMGB1 Transport}

To identify which of the six serine residues in the NLS 1 and 2 regions is critical for HMGB1 transport from the nucleus 
to the cytoplasm, we constructed six HMGB1 vectors (NLS35ET, NLS-39ET, NLS-42ET, NLS-46ET, NLS-53ET, and NLS-181ET), each containing a serine to glutamic acid mutation at the indicated residue (Figure 1a). We also constructed one vector (NLS(1)-ET) for which all five serine residues in the NLS1 region were mutated to glutamic acid. The matched vector NLS(1)-AT was also constructed, where all five serine residues were converted to alanine. In this transfection study, we observed that only cells transfected with NLS(1)-ET, NLS-35ET, NLS-39ET, and NLS-42ET showed increased HMGB1 transport from the nucleus to the cytoplasm as compared with cells transfected with the other three vectors (Figure 1c), suggesting that serine residues at 35,39 , and 42 are important for HMGB1 transport.

\section{HMGB1 Phosphorylation is Inhibited by Kinase Inhibitors}

To identify factors responsible for HMGB1 phosphorylation, we used the NetPhosK 1.0 server (http://www.cbs.dtu.dk/ services/NetPhosK/) to identify kinases that could potentially bind to and induce the phosphorylation of HMGB1. We identified casein kinase 1 (CK1) and PKC as possible candidates. Thereafter, we carried out a kinase inhibitor study to test which factor was involved in HMGB1 phosphorylation. We used Quercetin to inhibit CK1, and Tamoxifen, Myricetin, and Rottlerin to inhibit PKC. We also used Myricetin at both 3 and $10 \mu \mathrm{M}$, as it has been reported to inhibit CK1 or PKC at low or high concentrations, respectively. The distribution of HMGB1 did not change in HCT116 cells treated with Quercetin or with $3 \mu \mathrm{M}$ of Myricetin, indicating that CK1 was not involved in HMGB1 translocation. In contrast, HMGB1 expression in the cytoplasm was significantly decreased in cells treated with Tamoxifen and Rottlerin (Figure 5a). Decreased cytoplasmic expression of HMGB1 was also observed in cells treated with $10 \mu \mathrm{M}$ of Myricetin. The requirement for PKC was validated by immunoprecipitation analysis to test of co-interaction between $\mathrm{PKC}$ and HMGB1 (Figure 5b). These findings indicate that PKC signaling is involved in the cytoplasmic translocation of HMGB1 in colorectal cancer cells. a

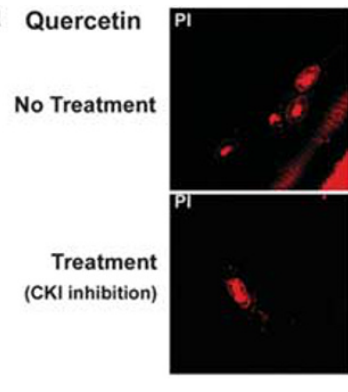

Myricetin

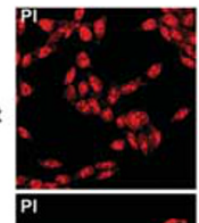

Treatment with

low dose

(CKI inhibition) Treatment with
high dose
(PKC inhibition)
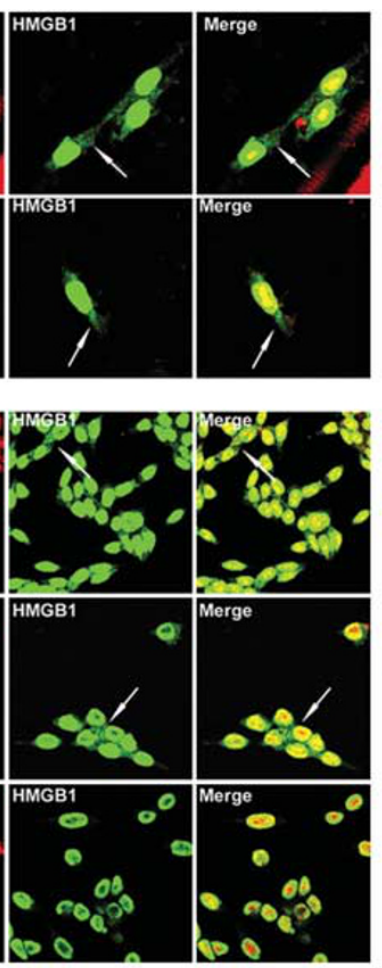
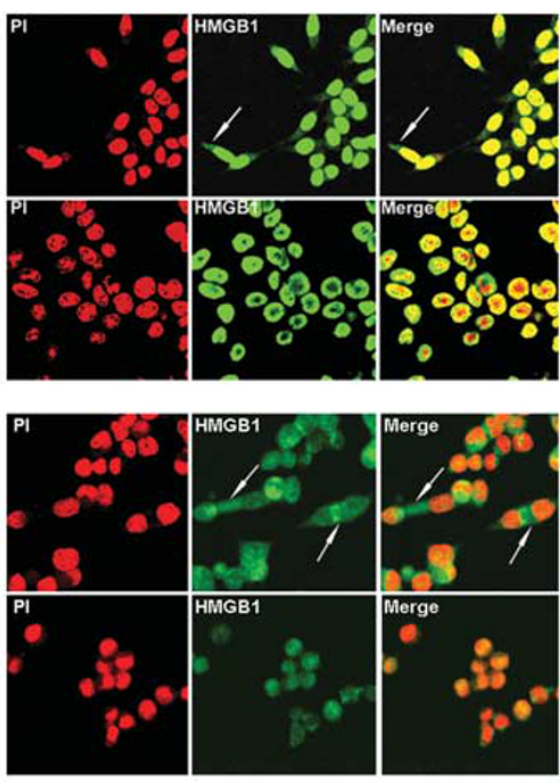

Tamoxifen

No Treatment
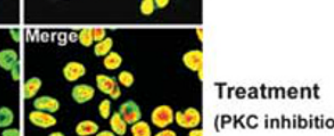

(PKC inhibition)

Rottlerin

No Treatment

Treatment

(PKC inhibition)

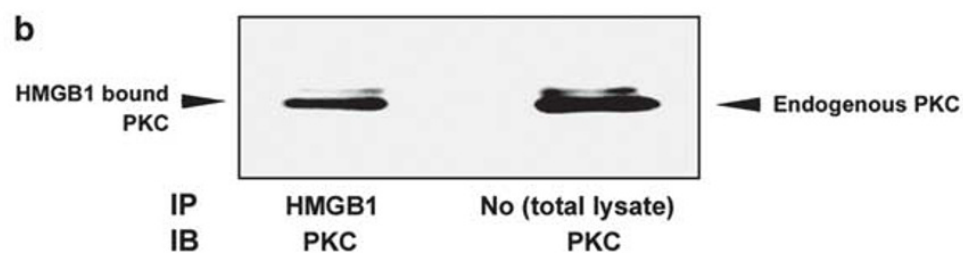

Figure 5 Cytoplasmic HMGB1 is decreased after treatment of kinase inhibitor. (a) The distribution of HMGB1 did not change with treatment of CK1 inhibitors, Quercetin or low dose ( $3 \mu \mathrm{M})$ of Myricetin treatment. HMGB1 expression in the cytoplasm is significantly decreased in treatment of PKC inhibitors, Tamoxifen, and Rottlerin treatment in HCT116 cells. Decreased cytoplasmic expression was also found in a high dose (10 $\mu \mathrm{M}$ ) of Myricetin treatment, one of PKC inhibitor. (b) Verification of the binding of PKC and HMGB1 by immunoprecipitation analysis in HCT116 cell line. Binding of PKC and HMGB1 was noted. 


\section{HMGB1 is Unidirectionally Transported from the Nucleus to the Cytoplasm}

HMGB1 is a small molecule and has the potential to migrate from the nucleus to the cytoplasm by simple diffusion across nuclear pores. To test whether the translocation of HMGB1 from the nucleus to the cytoplasm is unidirectional in a CRM1-dependent manner, or whether it is bidirectional by simple diffusion, we treated HCT116 cells with Leptomycine $\mathrm{B}$, an inhibitor of CRM1-mediated active export. We found that endogenous cytoplasmic HMGB1 was decreased markedly after treatment of Leptomycine $\mathrm{B}$, indicating that the cytoplasmic translocation of HMGB1 was unidirectional and conducted through active transport (data not shown).

\section{Secretion of HMGB1 is Related to Increased Tumor Cell Invasiveness}

The results described above predict that modified HMGB1 is secreted into the extracellular space. To confirm this idea, we measured the amount of HMGB1 by immunoblot in the culture medium from cells transfected with the vectors described above. A relatively large amount of HMGB1 was detected in the culture medium of HCT116 cells transfected with the NLS-WT or NLS(1,2)-ET vector. In contrast, scant amounts of HMGB1 were noted in the medium from cells transfected with the NLS(1,2)-AT vector (Figure 6a). To verify the phosphorylation status of HMGB1 in the culture medium, we performed immunoprecipitation experiments using media protein and an HMGB1 antibody, followed by immunoblot analysis using antibodies against phosphorylated proteins. We found that phosphorylated HMGB1 was present in the culture medium of cancer cells (Figure 6b). These findings indicate that phosphorylated HMGB1 is transported to the cytoplasm and subsequently secreted into the extracellular space.

To extend these observations to tumor cell invasiveness, we next performed the invasion assay using tissue culture plate containing an $8-\mu \mathrm{m}$ pore size polycarbonate membrane, which blocked non-invasive cells from migrating through. Invasive cells, on the other hand, migrate through the layer and cling to the bottom of the polycarbonate membrane. We found that the invasiveness was increased markedly in cells transfected with NLS(1,2)-ET and slightly increased in cells transfected with NLS-WT. Cells transfected with NLS $(1,2)$ AT or the control vector showed low invasion activity compared with cells transfected with NLS-WT or NLS(1,2)-ET (Figure 6c). Especially, we observed that the invasiveness was increased in cells transfected with NLS(1)-ET, NLS-35ET, NLS-39ET, and NLS-42ET as compared with cells transfected with the other three vectors (Figure 6c). To ascertain the role of secreted HMGB1, we treated cells with recombinant HMGB1 by including it in the culture medium, and found that invasiveness was increased markedly in HCT116 cells (Figure 6d). These findings indicate that phosphorylated HMGB1 is directly related to cell invasion capacity. We also constructed one vector (NLS(1)-ET) in which all five serine residues in the NLS1 region were mutated to glutamic acid. The matched vector NLS(1)-AT was also constructed, where all five serine residues were converted to alanine. In this transfection study, we observed that only cells transfected with NLS(1)-ET, NLS-35ET, NLS-39ET, and NLS-42ET showed increased HMGB1 transport from the nucleus to the cytoplasm as compared with cells transfected with the other three vectors (Figure 1c), suggesting that serine residues at 35, 39, and 42 are important for HMGB1 transport.

\section{DISCUSSION}

In this study, we showed the overexpression of HMGB1 in cancer cells, cytoplasmic transport of HMGB1, the presence of extracellular HMGB1, HMGB1 phosphorylation, activation of the MMP pathway, and increased cell invasion after transfection of activated HMGB1. These findings suggest that HMGB1 indeed has a role in the ability of cancer cells to invade surrounding tissue and metastasis.

One of the important roles of HMGB1 is cell migration, and metastasis in cancer cells involves the activation of RAGE and the MMP pathway. ${ }^{2,12,18,19}$ It has been proposed that secreted HMGB1 from both inflammatory and necrotic cells has a role in this signal transduction event. ${ }^{19-23}$ In this study, we showed that endogenous HMGB1 can be secreted into the extracellular space, where it can bind to RAGE and activate the MMP pathway. Our novel finding that endogenous HMGB1 can cause aberrant signaling events in cancer cells partially explains the early phase of cancer metastasis, which is a complicated and multistep process. Little is known about the mechanism by which cancer cells acquire invasion and cell migration ability. In this study, we suggest that the signals required for metastasis can be initiated by the cancer cell itself through autocrine and/or paracrine activation by the secreted HMGB1.

Our data suggest that the nuclear and/or cytosolic distribution of HMGB1 is caused by protein phosphorylation. We propose that the cytoplasmic transport of HMGB1 is the result of its phosphorylation. It has been shown that phosphorylation of HMGB1 leads to more negatively charged groups on the protein, reducing its DNA-binding activity by up to 10 -fold. ${ }^{24,25}$ Therefore, we suggest that the reduced DNA-binding affinity of HMGB1 might be related to its cytoplasmic transport. We modified the serine residues in two NLS regions of HMGB1 to glutamic acid (NLS(1,2)-ET) for simulating phosphorylation and to alanine $\operatorname{NLS}(1,2)$ AT) for preventing phosphorylation. Cells transfected with the NLS(1,2)-ET vector showed increased cytoplasmic HMGB1 transport, whereas HMGB1 was retained in the nucleus in cells transfected with the NLS(1,2)-AT vector. These findings indicate that the negatively charged status of HMGB1 was directly related to cytoplasmic transport. Moreover, our CRM1 inhibition study showed a marked reduction of endogenous cytoplasmic HMGB1. This indicates that HMGB1 is translocated from the nucleus to the cytoplasm by active transport rather than by simple diffusion, 
a
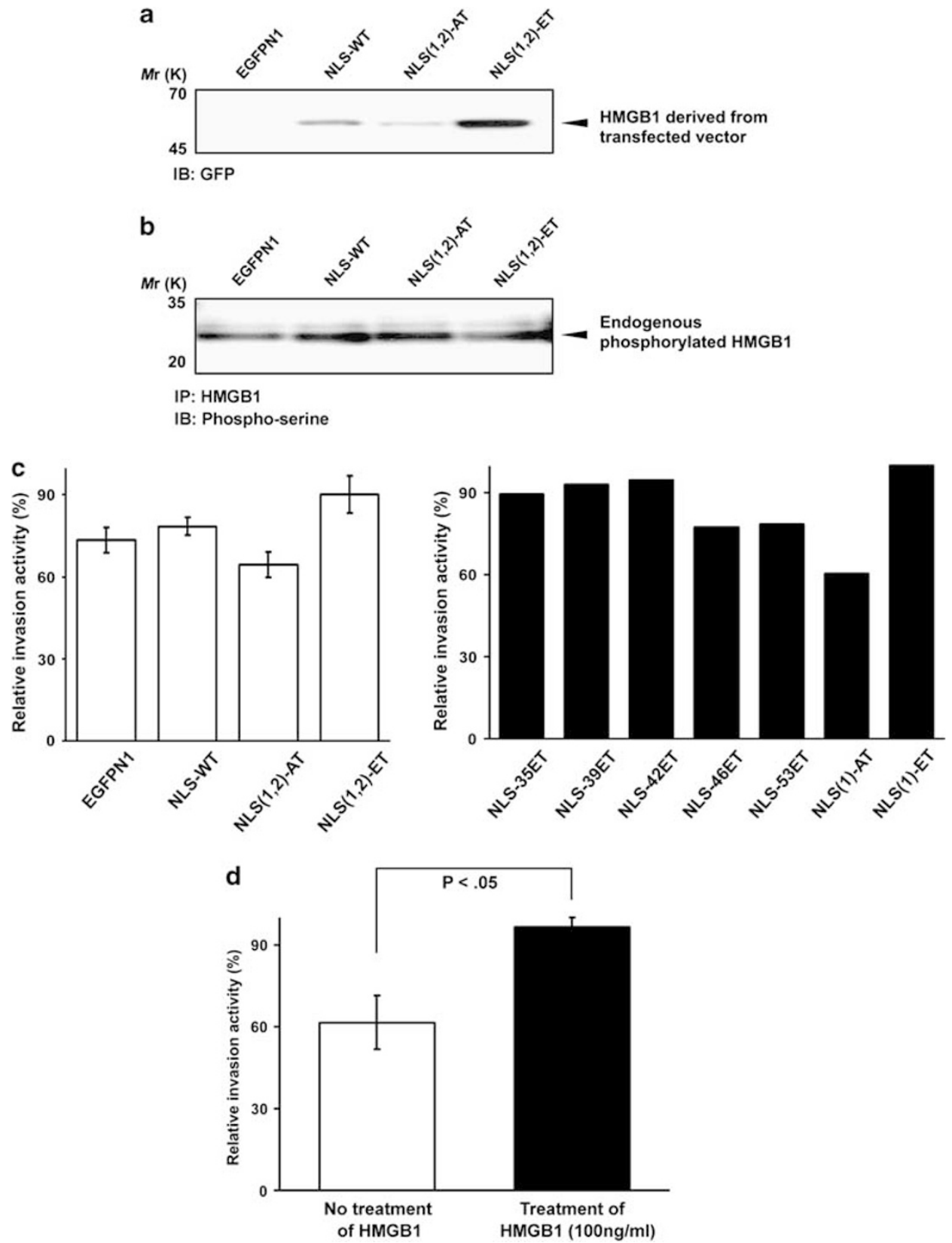

Figure 6 Extracellular HMGB1 promote cell invasion. (a) Immunoblot analysis of HMGB1 from the culture medium of HCT116 cells transfected with NLS-WT, NLS(1,2)-AT, or NLS(1,2)-ET vectors. Secreted HMGB1 was evident in the cell lines transfected with NLS-WT and NLS(1,2)-ET. (b) Immunoprecipitation of media protein lysates using HMGB1 antibody and immunoblot using antibodies against phosphorylated proteins. Similar amount of endogenous phosphorylated HMGB1 was found in all of four cell lines transfected with different vectors. (c) Invasion activity are increased in HCT116 cells transfected with NLS(1,2)-ET vector than in cells transfected with other forms of vectors (NLS-WT, NLS(1,2)-AT and EGFPN1). Especially, the invasiveness was increased in cells transfected with NLS(1)-ET, NLS-35ET, NLS-39ET, and NLS-42ET as compared with cells transfected with the other three vectors (NLS-46ET, NLS-53ET, and NLS(1)-AT). (d) Treatment of recombinant HMGB1 protein in the culture media showed increased invasiveness of tumor cells.

and implies that HMGB1 phosphorylation occurs in the nucleus. These findings also suggest that a signal transduction pathway activated in these cancer cells regulates the translocation of endogenous HMGB1.

Our results suggest that HMGB1 phosphorylation might be induced by PKC. In addition, the phosphorylation of serines 35, 39, and 42 of the NLS1 region in HMGB1 is critical for HMGB1 transport into the cytoplasm, and these serine residues are consistent with the predicted PKC binding site. The PKC family consists of at least 12 serine-threonine kinases that are classified into three major groups, ${ }^{26,27}$ and their expression level was variable according to cancer type. The most important and well-known cancer-related targets of PKC are Erk1/2, glycogen synthase kinase-3 beta (GSK-3 $\beta$ ), 
nuclear factor kappa B (NFkB), and P-glycoprotein. ${ }^{28,29}$ Previous reports have shown that the biological functions of PKC are mostly linked to events occurring at the plasma membrane or in the cytoplasm. PKC isoforms are thought to reside in the cytoplasm in an inactive state, translocated to the plasma membrane after stimulation, and become activated in the presence of specific co-factors. ${ }^{30-33}$ However, some reports have indicated that PKC isoforms are located in the nucleus or are translocated to the nucleus. Similarly, other reports have suggested a direct relationship between nuclear PKC and tumorigenesis, ${ }^{34-37}$ although the regulatory role of nuclear PKC in cancer is not completely understood. ${ }^{34-39}$ In this study, we showed that HMGB1 interacted with PKC. Future functional analysis for the relationship between the specific type of PKC and nuclear phosphorylation might provide valuable information about the role of HMGB1 in tumor progression and invasion. In addition, HMGB1 indeed has a 'double-edged role,' such as interacting with infiltrating RAGE expressing monocytes and lymphocytes, besides possibly activating other innate receptors, TLR4 and TLR2. ${ }^{40-43}$

In conclusion, we showed that a portion of nuclear HMGB1 is phosphorylated in colon cancers and that phosphorylated HMGB1 is believed to be involved in the extracellular secretion. Further study is required to elucidate that the secreted HMGB1 activates the genes involved in tumor invasion and migration of cancer cells. Phosphorylated HMGB1 then induces migration of the cancer cells. These findings suggest that cancer cells can acquire the ability to invade surrounding tissues and to metastasize through the activation of endogenous nuclear HMGB1.

\section{ACKNOWLEDGEMENT}

This study was supported by a grant of the Korea Health 21 R\&D Project (Ministry of Health \& Welfare (03-PJ10-PG6-GP01-0002)) and FPR08A2-100 of the 21C Frontier Functional Proteomics Project from the Korean Ministry of Science \& Technology.

1. Bonaldi T, Langst $G$, Strohner $R$, et al. The DNA chaperone HMGB facilitates ACF/CHRAC-dependent nucleosome sliding. EMBO J 2002;21:6865-6873.

2. Pallier C, Scaffidi P, Chopineau-Proust $S$, et al. Association of chromatin proteins high mobility group box (HMGB) 1 and HMGB2 with mitotic chromosomes. Mol Biol Cell 2003;14:3414-3426.

3. Stros M, Ozaki T, Bacikova A, et al. HMGB1 and HMGB2 cell-specifically down-regulate the p53- and p73-dependent sequence-specific transactivation from the human Bax gene promoter. J Biol Chem 2002;277:7157-7164.

4. Fages $\mathrm{C}$, Nolo $\mathrm{R}$, Huttunen $\mathrm{HJ}$, et al. Regulation of cell migration by amphoterin. J Cell Sci 2000;113(Pt 4):611-620.

5. Lotze MT, Tracey KJ. High-mobility group box 1 protein (HMGB1): nuclear weapon in the immune arsenal. Nat Rev Immunol 2005;5: 331-342.

6. Muller S, Scaffidi P, Degryse B, et al. New EMBO members' review: the double life of HMGB1 chromatin protein: architectural factor and extracellular signal. EMBO J 2001;20:4337-4340.

7. Taguchi A, Blood DC, del Toro G, et al. Blockade of RAGE-amphoterin signalling suppresses tumour growth and metastases. Nature 2000;405:354-360.

8. Travers AA. Priming the nucleosome: a role for HMGB proteins? EMBO Rep 2003;4:131-136.
9. Ito $\mathrm{T}$, Kawahara K, Nakamura $\mathrm{T}$, et al. High-mobility group box 1 protein promotes development of microvascular thrombosis in rats. J Thromb Haemost 2007;5:109-116.

10. Gardella $S$, Andrei $C$, Ferrera D, et al. The nuclear protein HMGB1 is secreted by monocytes via a non-classical, vesicle-mediated secretory pathway. EMBO Rep 2002;3:995-1001.

11. Bonaldi T, Talamo F, Scaffidi $P$, et al. Monocytic cells hyperacetylate chromatin protein HMGB1 to redirect it towards secretion. EMBO J 2003;22:5551-5560.

12. Taguchi A, Blood DC, del Toro G, et al. Blockade of amphoterinreceptor for age (RAGE) interaction suppresses lung metastasis in murine Lewis lung carcinoma. FASEB J 1999;13:A363.

13. Muller S, Ronfani L, Bianchi ME. Regulated expression and subcellular localization of HMGB1, a chromatin protein with a cytokine function. J Intern Med 2004;255:332-343.

14. Choi YR, Kim H, Kang HJ, et al. Overexpression of high mobility group box 1 in gastrointestinal stromal tumors with KIT mutation. Cancer Res 2003;63:2188-2193.

15. Volp K, Brezniceanu ML, Bosser S, et al. Increased expression of high mobility group box 1 (HMGB1) is associated with an elevated level of the antiapoptotic c-IAP2 protein in human colon carcinomas. Gut 2006;55:234-242.

16. Kang $\mathrm{HJ}$, Koh KH, Yang $\mathrm{E}$, et al. Differentially expressed proteins in gastrointestinal stromal tumors with KIT and PDGFRA mutations. Proteomics 2006;6:1151-1157.

17. Youn JH, Shin JS. Nucleocytoplasmic shuttling of HMGB1 is regulated by phosphorylation that redirects it toward secretion. J Immunol 2006;177:7889-7897.

18. Dumitriu IE, Baruah $\mathrm{P}$, Bianchi $\mathrm{ME}$, et al. Requirement of HMGB1 and RAGE for the maturation of human plasmacytoid dendritic cells. Eur J Immunol 2005;35:2184-2190.

19. Dumitriu IE, Baruah $P$, Valentinis $B$, et al. Release of high mobility group box 1 by dendritic cells controls $T$ cell activation via the receptor for advanced glycation end products. J Immunol 2005;174:7506-7515.

20. Raucci A, Palumbo R, Bianchi ME. HMGB1: a signal of necrosis. Autoimmunity 2007;40:285-289.

21. Fiuza C, Bustin M, Talwar S, et al. Inflammation-promoting activity of HMGB1 on human microvascular endothelial cells. Blood 2003;101:2652-2660.

22. Orlova VV, Choi EY, Xie C, et al. A novel pathway of HMGB1-mediated inflammatory cell recruitment that requires Mac-1-integrin. EMBO J 2007:26:1129-1139.

23. Scaffidi $P$, Misteli T, Bianchi ME. Release of chromatin protein HMGB1 by necrotic cells triggers inflammation. Nature 2002;418:191-195.

24. Ito I, Mitsuoka N, Sobajima J, et al. Conformational difference in HMGB1 proteins of human neutrophils and lymphocytes revealed by epitope mapping of a monoclonal antibody. J Biochem 2004;136: 155-162.

25. Stemmer C, Schwander A, Bauw G, et al. Protein kinase CK2 differentially phosphorylates maize chromosomal high mobility group B (HMGB) proteins modulating their stability and DNA interactions. J Biol Chem 2002;277:1092-1098.

26. Nishizuka Y. Intracellular signaling by hydrolysis of phospholipids and activation of protein kinase C. Science 1992;258:607-614.

27. Paolucci L, Rozengurt E. Protein kinase $D$ in small cell lung cancer cells: rapid activation through protein kinase C. Cancer Res 1999;59: 572-577.

28. Burgering BM, de Vries-Smits $\mathrm{AM}$, Medema $\mathrm{RH}$, et al. Epidermal growth factor induces phosphorylation of extracellular signalregulated kinase 2 via multiple pathways. Mol Cell Biol 1993;13: $7248-7256$.

29. Goode N, Hughes K, Woodgett JR, et al. Differential regulation of glycogen synthase kinase-3 beta by protein kinase $C$ isotypes. J Biol Chem 1992;267:16878-16882.

30. Hug $\mathrm{H}$, Sarre TF. Protein kinase $\mathrm{C}$ isoenzymes: divergence in signal transduction? Biochem J 1993;291(Pt 2):329-343.

31. Newton AC. Regulation of protein kinase C. Curr Opin Cell Biol 1997;9:161-167.

32. Newton AC. Regulation of protein kinase $C^{\prime}$ 's membrane interaction by two membrane-targeting domains? Biophys J 1997;72:Tup88.

33. Keranen LM, Newton AC. Ca2+ differentially regulates conventional protein kinase $\mathrm{Cs}^{\prime}$ membrane interaction and activation. J Biol Chem 1997;272:25959-25967. 
34. Cambier JC, Newell MK, Justement LB, et al. la binding ligands and CAMP stimulate nuclear translocation of PKC in B lymphocytes. Nature 1987;327:629-632.

35. Filomenko R, Poirson-Bichat F, Billerey C, et al. Atypical protein kinase $C$ zeta as a target for chemosensitization of tumor cells. Cancer Res 2002;62:1815-1821.

36. Malviya AN, Block C. Nuclear protein kinase $C$ and signal transduction. Receptor 1993;3:257-275.

37. La Porta CA, Dolfini E, Comolli R. Inhibition of protein kinase C-alpha isoform enhances the P-glycoprotein expression and the survival of LoVo human colon adenocarcinoma cells to doxorubicin exposure. Br J Cancer 1998;78:1283-1287.

38. Lu Y, Jamieson L, Brasier AR, et al. NF-kappaB/RelA transactivation is required for atypical protein kinase $C$ iota-mediated cell survival. Oncogene 2001;20:4777-4792.
39. Greif $\mathrm{H}$, Ben-Chaim J, Shimon $\mathrm{T}$, et al. The protein kinase C-related PKC-L(eta) gene product is localized in the cell nucleus. Mol Cell Biol 1992;12:1304-1311.

40. Park JS, Svetkauskaite D, He Q, et al. Involvement of toll-like receptors 2 and 4 in cellular activation by high mobility group box 1 protein. J Biol Chem 2004;279:7370-7377.

41. Yu $M$, Wang $H$, Ding $A$, et al. HMGB1 signals through toll-like receptor (TLR) 4 and TLR2. Shock 2006;26:174-179.

42. Park JS, Gamboni-Robertson F, He QB, et al. High mobility group box 1 protein interacts with multiple toll-like receptors. Am J Physiol-Cell Physiol 2006;290:C917-C924.

43. van Beijnum JR, Buurman WA, Griffioen AW. Convergence and amplification of toll-like receptor (TLR) and receptor for advanced glycation end products (RAGE) signaling pathways via high mobility group B1 (HMGB1). Angiogenesis 2008;11:91-99.

Supplementary Information accompanies the paper on the Laboratory Investigation website (http://www.laboratoryinvestigation.org) 